\title{
Orientação Psicológica On-line: Percepção dos Profissionais sobre a Relação com os Clientes
}

Online Psychological Counseling: the Perception of Professionals about the Relationship with Clients

Orientación Psicológica Online: Percepción de los Profesionales sobre la Relación con los Clientes

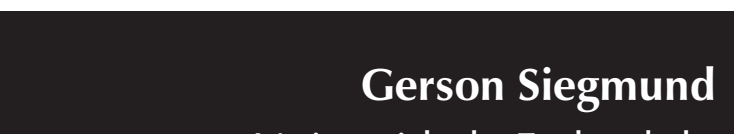

Universidade Federal do

Rio Grande do Sul

Carolina Lisboa

Pontifícia Universidade Católica do

Rio Grande do Sul

http://dx.doi.org/10.1590/1982-3703001312012 
Resumo: Os atendimentos psicológicos pela internet são uma prática recente, que vem recebendo crescente atenção de profissionais e pesquisadores. Há poucos estudos sobre o tema no Brasil, sendo necessário investigá-lo e compreendê-lo. A resolução 012/2005 do Conselho Federal de Psicologia regulamenta os serviços psicológicos mediados por computador, entre os quais destacase a orientação psicológica on-line. O presente estudo, de delineamento qualitativo e exploratório, teve por objetivo investigar a percepção dos profissionais acerca da orientação psicológica on-line, com foco na relação estabelecida com os clientes. Participaram da pesquisa quatro profissionais, homens e mulheres, com diferentes idades, práticas e tempo de atuação profissional. Para coleta dos dados foram utilizadas entrevistas on-line individuais semiestruturadas. Os participantes foram selecionados a partir de sites de atendimento on-line regulamentados pelo CFP. A análise dos dados foi feita através da análise de conteúdo de Bardin. Em relação aos resultados, foi possível perceber que essa prática possui limites e potencialidades. Conclui-se que o vínculo estabelecido nessa relação é diferenciado, mas possibilita a realização dos objetivos do atendimento e o acolhimento do cliente. Os atendimentos pela internet constituem uma prática nova, que necessita ser estudada, a fim de gerar maior entendimento e esclarecimento na área, para profissionais e clientes.

Palavras-chave: Orientação psicológica on-line. Relação profissional-cliente. Atendimentos psicológicos pela internet. Psicologia on-line.

Abstract: Psychological online treatments are a recent practice which has been receiving growing attention from professionals and researchers. There are few studies on this subject in Brazil, and further investigation to better understand it is needed. Resolution 012/2005 of the Federal Council of Psychology regularizes the computer-mediated psychological services, among which online counseling is highlighted. Using a qualitative and exploratory design, the present study aimed to investigate the perception of professionals about online counseling, focusing on the relationship established with clients. Four professionals, men and women, of different ages, practices and time of professional exercise were interviewed. Semi-structured online individual interviews were used for data collection. Participants were chosen from regularized sites registered at the Federal Council of Psychology. Content analysis of Bardin was carried out for data analysis. Regarding the study results, it was possible to observe that this practice presents both limits and potentialities. We concluded that the bond established in this relationship is different from that observed in regular clinical practice, but it allows for achievement of treatment goals and fulfillment of clients' expectancies. Online treatments constitute a new practice which needs to be studied in order to generate greater understanding and enlightenment to the area, representing benefits for both professionals and clients.

Keywords: Online counseling. Professional-client relationship. Psychological treatment over the Internet. Psychology online.

Resumen: Los tratamientos psicológicos por Internet son una práctica reciente, que ha sido objeto de una atención cada vez mayor por parte de profesionales e investigadores. Existen pocos estudios sobre este tema en Brasil, por lo tanto es necesario investigarlo y comprenderlo. Por Resolución 012/2005 del Consejo Federal de Psicología se regulan los servicios psicológicos mediados por ordenador, entre los que destaca el asesoramiento psicológico online. El presente estudio, de diseño cualitativo y exploratorio, tuvo como objetivo investigar las percepciones de los profesionales sobre el asesoramiento psicológico online, centrándose en la relación establecida con los clientes. Los participantes fueron cuatro profesionales, hombres y mujeres, de diferentes edades, prácticas y tiempo de actuación profesional. Para la recolección de datos, se usaron entrevistas individuales online, semi-estructuradas. Los participantes fueron seleccionados de entre sites de tratamiento online regulados por el Consejo Federal de Psicología. El análisis de datos se realizó mediante análisis de contenido de Bardin. En cuanto a los resultados, se reveló que esta práctica tiene límites y potencialidades. Se concluye que el vínculo que se establece en esta relación es diferente, pero permite la realización de los objetivos del tratamiento y la acogida del cliente. Los tratamientos por Internet son una nueva práctica, que debe ser estudiada con el fin de generar un mayor entendimiento y aclaración en el área de conocimiento, para profesionales y clientes.

Palabras-clave: Asesoramiento psicológico online. Relación profesional-cliente. Tratamiento psicológico por Internet. Psicología online. 


\section{Introdução}

A informatização e o uso da internet vêm crescendo visivelmente na última década. Segundo relatórios divulgados pela Organização das Nações Unidas (ONU, 2009), o número de pessoas com acesso à internet no mundo aumentou exponencialmente entre os anos de 2000 e 2008, e o Brasil acompanhou esse crescimento. Atualmente, somos quase 78 milhões de pessoas com acesso a internet em diversos ambientes (IBOPE, 2011) e ultrapassamos a marca de 40 milhões de usuários ativos (ONU, 2011).

Há mais de uma década atrás, o filósofo Pierre Lévy escreveu sobre o desenvolvimento das novas tecnologias e sua influência sobre a subjetividade humana. Afirmou: "estamos vivendo a abertura de um novo espaço de comunicação, e cabe apenas a nós explorar as potencialidades mais positivas desse espaço nos planos econômico, político, cultural e humano" (Lévy, 2008, p. 11).

Tais informações indicam a necessidade de um olhar atento da Psicologia para esse novo campo de estudo, e mais recentemente, de prática dos psicólogos. Para Bastos e Gondim (2010), um dos grandes desafios na atuação do profissional psicólogo brasileiro é o crescente desenvolvimento das tecnologias de comunicação e informação. Muitas alterações têm ocorrido na sociedade à medida que a tecnologia progride, e essas mudanças demandam um processo de reformulação do mundo e da profissão do psicólogo (Malvezzi, 2010).

Segundo Nicolaci-da-Costa (2005), a internet pode servir como ferramenta para a prática da Psicologia. Portanto, vemos que a internet é um instrumento que pode ser utilizado para a informação, conexão social, educação e autoeducação, economia, enfrentamento da timidez e inibição, citando apenas alguns exemplos. Para algumas pessoas, ela pode se tornar um problema à medida que passam a depender dela. Para outras, a internet contribui para o bem-estar e qualidade de vida (Aboujaoude, 2011). Sendo assim, é importante investigar e explorar a utilização dessa ferramenta no campo da Psicologia.
Estamos vivendo em um período crucial na história da humanidade, um período em que é possível interagir em tempo real com o mundo, com as pessoas e com a informação. É difícil estabelecer o limite entre necessidade e dependência e, por esse motivo, faz-se necessário encontrar meios de utilizar a tecnologia de maneira produtiva. Comportamentos e costumes estão sofrendo alterações significativas e novas formas de relacionamento entre as pessoas surgem, mediadas pelo campo virtual. Devemos estar atentos para o fato de que talvez ainda não estejamos preparados para lidar com essas mudanças tão impactantes do uso da internet. Portanto, é necessário investir em pesquisas na área, a fim de conhecer e aprofundar o fenômeno, podendo assim, entender minimamente o futuro da tecnologia e suas influências no nosso modo de ver e viver no mundo (Young \& Abreu, 2011).

\section{Relação profissional-cliente e relação terapêutica}

A noção de que a relação terapêutica desempenha um papel fundamental no processo psicoterapêutico vem sido explorada ao longo das últimas décadas e tem recebido atenção significativa dos pesquisadores de psicoterapia (Safran, 2002). A relação terapêutica é definida como o veículo através do qual se processam os tratamentos psicoterápicos (Eizirik, Libermann \& Costa, 2008).

Para Rogers $(1975,1997)$, a percepção do paciente sobre a relação terapêutica detinha uma importância central na probabilidade de progresso terapêutico, configurando-se essa como uma relação única, que exige grande atenção. Da mesma forma, ao formular os preceitos fundamentais da Terapia Cognitiva, Beck, Rush, Shaw e Emery (1997) procuraram dar especial atenção ao relacionamento terapêutico, e afirmaram: "[...] o terapeuta, ao aplicar terapia cognitiva, está continuamente ativo e deliberadamente interagindo com o paciente" (Beck et al., 1997, p. 6).

Em estudos realizados sobre a eficácia das práticas psicoterápicas no tratamento de problemas por uso de drogas e álcool, por exemplo, Washton e Zweben (2009) verificaram 
que a habilidade dos terapeutas em evocar uma relação terapêutica adequada com seus pacientes, bem como de expressar empatia, influenciam fortemente o resultado positivo e a adesão ao tratamento. Freeman e Dattilio (1998) também sugerem que desenvolver uma relação de trabalho terapêutico é um prerrequisito de qualquer tratamento, sendo que, em alguns casos, pode levar um longo tempo para que seja devidamente estabelecida. Ao mesmo tempo, Safran (2002) alerta para a necessidade de buscar conhecer o cliente e a forma como ele entende as intervenções terapêuticas.

Atesta-se que a tecnologia progride de forma rápida e consistente, e a Psicologia, como ciência, tem buscado se colocar a par desse desenvolvimento tecnológico, inserindo-se e utilizando-se desse contexto, especialmente da informática e internet. Observa-se, também, que a relação terapêutica ocupa um lugar fundamentalmente importante nas psicoterapias presenciais. Entretanto, não se tem tanta clareza sobre os serviços que a Psicologia tem a oferecer através da internet, e que papel a relação entre cliente e profissional ocupa nessa modalidade de atendimento psicológico.

\section{A relação terapêutica e os atendimentos psicológicos on- line}

Uma das formas de inserção ou integração da Psicologia no contexto tecnológico da informática são os atendimentos psicológicos on-line, ou serviços mediados pelo computador, como também são conhecidos. As possibilidades dessa modalidade de atendimento são quase tão diversas quanto os atendimentos psicológicos presenciais. Porém nem todos são reconhecidos ou legalizados. A resolução 012/2005, estabelecida pelo Conselho Federal de Psicologia, regulamenta as práticas e serviços psicológicos mediados por computador (CFP, 2005).

Essa legislação determina que a psicoterapia virtual pode ser praticada apenas a nível experimental, desde que sejam contempladas algumas condições, tais como: fazer parte de projeto de pesquisa, ter aprovação de Comitê de Ética, ser gratuita, entre outras
(CFP, 2005). Entretanto, a mesma legislação reconhece como prática legal e passível de remuneração outros serviços psicológicos mediados por computador desde que possuam caráter pontual e informativo. Entre os serviços citados, encontra-se a orientação psicológica on-line. A investigação acerca da relação desenvolvida entre profissional e clientes dos serviços de orientação psicológica on-line é o foco principal deste estudo.

Sendo assim, é necessário sublinhar a distinção entre essa prática e a prática da psicoterapia on-line. Por definição, a orientação on-line possui um caráter pontual e informativo, tendo sua diferenciação principal no foco das mensagens: deve deter-se em um assunto ou tema específico, de conhecimento do profissional (Fortim, 2004). Caso o objetivo seja um atendimento presencial, o profissional deve encaminhar o cliente para esse tipo de atendimento. Ademais, o número de mensagens ou de informações trocadas é limitado, sendo que deve haver um cuidado para que não se estabeleça um vínculo semelhante ao de uma terapia, e para que não sejam feitas interpretações sobre a vida do cliente.

A demanda para os atendimentos mediados por computador ainda não é clara. Profissionais do Núcleo de Pesquisa de Psicologia e Informática - NPPI da PUC-SP realizaram uma pesquisa objetivando entender o crescente aumento da procura pelo serviço de orientação psicológica via e-mail oferecido pela instituição. A fim de compreender essa procura, utilizou-se um levantamento de dados do serviço. Os profissionais concluíram que não existe um perfil exclusivo de usuário desse tipo de atendimento. O tipo de demanda foi bastante diverso: vivências dolorosas, temas sobre sexualidade, luto, uso patológico da internet, transtornos psiquiátricos diversos foram os motivos mais encontrados. As variáveis que determinaram a busca do auxílio pela internet também foram diversas: anonimato, utilização do e-mail como catarse emocional, popularização da internet, entre outras (Fortim \& Cosentino, 2007).

Outra pesquisa, realizada através de um programa de orientação profissional (OP) on-line, com 851 adolescentes do Programa de Adolescentes Trabalhadores, do Banco do Brasil, em 2003 e 2004, obteve avaliação 
positiva em todas as turmas de OP on-line (Spaccaquerche, 2005). A participação nos fóruns foi significativa e relevante para o processo de orientação. Concluiu-se, então, que é possível desenvolver um serviço de orientação profissional on-line eficiente e que seja proveitoso para o orientando. A autora afirma que esse estudo foi relevante por demonstrar a possibilidade de desenvolver com sucesso, pela internet, um serviço que anteriormente só havia sido pensado presencialmente.

Reforçando as pesquisas na área, Prado e Meyer (2006) realizaram um estudo com o objetivo de verificar se seria possível atender pacientes em psicoterapia breve, assíncrona, via internet. O estudo objetivava, também, verificar se somente com as informações enviadas e recebidas por intermédio do computador seria possível formar e manter uma relação terapêutica com características semelhantes a das outras formas de psicoterapia. A partir dos resultados, verificou-se a formação e mantimento da relação terapêutica de forma semelhante às psicoterapias presenciais e mostrou-se que foi possível estabelecer, pela internet, um clima agradável e produtivo entre pacientes e terapeutas.

Apesar da incompletude e deficiências da orientação on-line, ela pode ser um instrumento de muita utilidade para as pessoas que procuram serviço psicológico mediado por computador (Fortim, 2004). Prado (2000) complementa a questão, afirmando que se as pesquisas forem desenvolvidas de forma adequada, a terapia on-line poderia contribuir muito para a Psicologia de um modo geral, assim como para a psicoterapia na sua forma tradicional. Aponta como principal fator da psicoterapia presencial a relação terapêutica, destacando que esse é um fator que está presente nas mais diversas formas de psicoterapia existentes, independentemente da abordagem teórica utilizada. Partindo desse argumento, Prado e Meyer (2006) enfatizam a necessidade de pesquisar a possibilidade da relação terapêutica nas terapias virtuais, sendo esse um primeiro passo para avaliar a terapia via internet e avançar no desenvolvimento dos serviços mediados por computador. Alguns países, como Canadá e Holanda, entre outros, já realizaram pesquisas empíricas e metanálises mostrando resultados positivos dos atendimentos on-line para diversos tipos de transtornos psiquiátricos, como ansiedade, depressão e fobias (van Bastelaar, Cuijpers, Pouwer, Riper \& Snoek, 2011; Bouchard et al., 2004; Spek et al., 2007).

Dessa forma, o presente estudo teve como objetivo investigar a percepção de profissionais a respeito do atendimento que realizam pela internet, especialmente no que diz respeito ao vínculo que é estabelecido. Além disso, também se buscou compreender quais as particularidades desse tipo de atendimento, seus pontos positivos e negativos, os principais motivos de procura dos clientes, entre outras características da prática.

\section{Método}

Delineamento

Esta pesquisa consistiu em um estudo qualitativo, de caráter exploratório, que procurou investigar a relação entre profissionais e clientes na orientação psicológica on-line síncrona, a partir da percepção dos profissionais.

\section{Participantes}

Participaram da pesquisa quatro profissionais, homens e mulheres, com idade entre 31 e 46 anos, de serviços de orientação psicológica on-line. Foram incluídos neste estudo apenas profissionais que atendiam de forma síncrona e que possuíam site validado e aprovado pelo Conselho Federal de Psicologia, conforme a legislação. O tempo de atuação dos profissionais no contexto presencial variou entre 6 meses e 12 anos e as áreas de atuação eram diversas. O tempo de atuação no contexto on-line situou-se entre 4 meses e um ano. Cabe ressaltar que um dos profissionais possuía deficiência visual e utilizava um software que transformava as palavras escritas em áudio. As orientações teóricas utilizadas pelos entrevistados variaram entre cognitivo-comportamental, psicoterapia corporal neorreichiana, e abordagem centrada na pessoa. As modalidades de atendimento on-line disponibilizadas por esses profissionais são principalmente e-mail e MSN, mas também chat e Skype. 


\section{Instrumentos}

Foi realizada uma entrevista on-line semiestruturada com os profissionais, através de chat, com perguntas norteadoras sobre a prática da orientação psicológica on-line e a percepção da relação terapêutica com seus clientes. A duração das entrevistas foi de aproximadamente 60 minutos com cada profissional.

Procedimentos de pesquisa e procedimentos éticos

Para encontrar os participantes realizou-se uma busca através da lista de sites credenciados pelo Conselho Federal de Psicologia até maio de 2011, disponível na página virtual do conselho (http://selo.cfp.org.br/credenciamento/ siteAprovado.cfm). A partir disso, visitaramse as páginas dos profissionais para realizar contato por e-mail. O critério inicial de busca foi por tempo de atendimento/cadastro: os sites que estavam registrados há mais tempo no CFP foram os primeiros com os quais se fez contato. Um e-mail de apresentação do pesquisador e da pesquisa foi enviado, solicitando retorno caso o profissional desejasse participar. Conforme o fluxo de respostas, esse procedimento foi realizado com todos os sites da lista. No total, 14 profissionais responderam o e-mail. Porém, três deles recusaram-se a participar da pesquisa, dois não puderam ser incluídos porque faziam apenas atendimentos assíncronos, dois não retornaram contato posterior e três ainda não haviam realizado atendimentos on-line, apesar de terem o site registrado. Houve, também, uma tentativa de contato com o NPPI (Núcleo de Pesquisa em Psicologia e Informática da PUC-SP), porém nesse serviço os profissionais realizam apenas atendimentos assíncronos.

Os participantes obtiveram orientações e esclarecimentos sobre a pesquisa e receberam o Termo de Consentimento Livre e Esclarecido por e-mail. Assim, tinham três opções: a) assinar manualmente e enviar de volta por e-mail; b) assinar manualmente e enviar por correio; ou c) utilizar assinatura digital identificada. Após recebimento do TCLE assinado, agendou-se a data e hora do chat com cada participante. As entrevistas foram realizadas sincronicamente, por intermédio do computador, através de um chat simples, criado unicamente para o propósito desta pesquisa. A conversa realizada no chat foi salva e convertida em arquivo do Word, para análise posterior. Os registros no site foram deletados e o domínio desfeito.

Antes de colocado em prática, o projeto de pesquisa foi encaminhado e aprovado pelo Comitê de Ética em Pesquisa da Universidade do Vale do Rio dos Sinos - UNISINOS, n. CEP 11/104, e seguiu as recomendações éticas para pesquisas com seres humanos conforme a resolução 196/1996 do Conselho Nacional de Saúde - CNS. Foi prevista uma devolução dos dados aos participantes que demonstraram interesse.

Análise dos dados

Para análise dos dados, foi utilizada a análise de conteúdo, seguindo-se os passos de préanálise, exploração do material e tratamento dos resultados, conforme descrito por Bardin (1995). O material foi dividido em categorias a posteriori, sendo essas agrupadas por proximidade de conteúdo, conforme apresentado a seguir. A fim de permitir que o leitor visualize como foi realizada a divisão, exemplos de fala são citados como ilustração para cada categoria.

Motivações: incluíram-se nessa categoria as respostas que se referiam às motivações mais frequentemente identificadas na busca dos pacientes pelo atendimento on-line. Exemplo: "Na minha experiência, procuraram mais por transtorno de humor".

Percepções acerca da própria atividade: incluíram-se nessa categoria as respostas relativas à quantidade de demanda e ao nível de dedicação do profissional a essa atividade. Exemplo: "Na verdade fiz poucas orientações, ou melhor, tive poucos orientados, não é minha única atividade".

Características do atendimento: incluíram-se nessa categoria as respostas referentes às características do atendimento. Exemplo: "De acordo com orientação do CFP, orientação psicológica on-line não pode ultrapassar 10 atendimentos".

Atendimento presencial $\mathrm{x}$ atendimento pela internet: incluíram-se nessa categoria 
as respostas relativas às percepções acerca dos encaminhamentos para atendimento presencial e da preferência por ele. Exemplo: "Eu sempre encaminho para terapia presencial, falo da importância do contato olho no olho, do sentir".

Pontos positivos: incluíram-se nessa categoria os pontos que os profissionais declararam como positivos nos atendimentos pela internet. Exemplo: "Se quiser dizer uma facilidade para o profissional, é ele poder ler muitas vezes antes de dar a resposta para a pessoa".

Pontos negativos: incluíram-se nessa categoria os pontos que os profissionais declararam como negativos nos atendimentos pela internet. Exemplo: "O orientado precisa estar totalmente envolvido com a sessão, querer mesmo, porque enquanto nos falamos, ele pode ficar navegando em outros sites e você nem perceber".

Estabelecimento de vínculo: incluíram-se nessa categoria as respostas relacionadas ao estabelecimento de vínculo com os clientes pela internet. Exemplo: "É um vínculo virtual, mas é um tipo de relação, vínculo não é só presencial, vínculo pode existir independente de ser presencial ou on-line".

Expressão de empatia: incluíram-se nessa categoria as respostas relativas a como eles percebem a expressão de empatia e como acreditam que isso é percebido pelos clientes. Exemplo: "Percebo que a relação com o orientando on-line é tão empática quanto no atendimento convencional, com uma ressalva: é necessário mais atenção ao que se fala e escreve, do que ao que se visualiza, uma vez que essa visualização nem sempre é clara e precisa (audiovisuais podem não apresentar as expressões claras do corpo)".

Resposta a objetivos: incluíram-se nessa categoria as respostas relacionadas ao cumprimento dos objetivos propostos para o atendimento on-line. Exemplo: "Lembro muito bem de um caso: (...) sofria de anorexia. Depois de uns três meses de trocas de e-mails, conseguiu buscar ajuda na sua cidade. Hoje está bem, continua em terapia, se conhecendo, descobrindo-se".
Outros: incluíram-se nessa categoria falas e respostas que não constituíam uma nova categoria, nem foram classificadas em nenhuma das demais. Exemplo: "Sabe que eu sinto que cada vez mais as pessoas estão se ausentando do contato vivo e isso na verdade é muito triste".

\section{Resultados e discussão}

Percebe-se, segundo as falas dos participantes, que as motivações que trazem os clientes para o atendimento on-line são bastante diversas, alinhavando-se aos resultados do levantamento de demanda realizada por Fortim e Cosentino (2007) no Núcleo de Pesquisa em Psicologia e Informática (NPPI) da PUC-SP. Alguns motivos de busca que se assemelharam nos dois serviços foram: conteúdos relativos à orientação sexual e afetiva, conflitos familiares, conflitos interpessoais, transtornos de humor e transtornos alimentares. A busca por orientação profissional foi citada na presente pesquisa e não apareceu na pesquisa do NPPI. Diversos outros conteúdos levantados no NPPI não foram citados pelos profissionais da presente pesquisa. Pode-se atribuir essa diferença às características de atendimento dos diferentes serviços, bem como à diferença na quantidade da demanda.

Analisando-se as categorias que dizem respeito à atividade profissional, é interessante observar que, para os profissionais entrevistados, a orientação psicológica pela internet constitui uma forma de trabalho secundária, complementar à sua atividade de trabalho principal, cuja preferência geral é a clínica presencial. A atuação em mais de uma área está em consonância com a realidade do profissional no Brasil, que atua concomitantemente em diferentes áreas, prestando serviços diversos (Heloani, Macêdo \& Cassiolato, 2010), tendo como causa principal a baixa remuneração na categoria.

Os participantes do presente estudo também fizeram referência a uma baixa quantidade de clientes e certa preferência pelo atendimento "ao vivo". Tais colocações podem se dar porque a prática dos atendimentos pela internet é relativamente recente e mais recente ainda é a sua regulamentação pelo Conselho Federal de Psicologia, que ocorreu em 2005 (CFP, 2005), sendo desconhecida por leigos e 
até mesmo profissionais da área. Dessa forma, é esperado que ainda se observe significativa baixa frequência na procura desse tipo de serviço. Além disso, podemos pensar que, por se tratar de uma atividade complementar para esses profissionais, não carrega a mesma implicação e investimento, hipótese que pode ser fortalecida pela fala de um dos participantes, quando diz que não investiu muito na divulgação do seu site. Também convém salientar que preconceitos acerca do atendimento on-line e das novas práticas psicológicas podem interferir na procura.

Por outro lado, existe uma questão fundamental, que amplia a discussão acima: a despeito do trabalho pela internet, a preferência dos profissionais entrevistados é pelo atendimento clínico presencial. Atualmente, de acordo com estudos realizados em âmbito nacional com profissionais (Bastos \& Gomide, 1989; Gondim, Bastos \& Peixoto, 2010) e estudantes de psicologia (Magalhães, Straliotto, Keller \& Gomes, 2001), o modelo hegemônico na Psicologia é o do psicólogo clínico tradicional, cuja área possui a maior concentração de profissionais e desperta mais fascínio entre os estudantes. Os locais de trabalho mais utilizados são a clínica e os consultórios particulares e a principal atividade realizada pelos psicólogos clínicos é, ainda, a psicoterapia individual. Dessa forma, não surpreende que os profissionais entrevistados tenham preferência pelo atendimento presencial tradicional. Contudo, essa preferência também pode ser um reflexo do caráter limitado da orientação psicológica on-line, sendo muito diferente do que acontece em uma psicoterapia, onde se permite um processo mais duradouro, trabalhando-se diversos aspectos da vida do sujeito. Se esse mesmo estudo fosse realizado com profissionais que realizassem psicoterapia on-line, ao invés de apenas orientação, o resultado seria possivelmente diferente.

Outro ponto importante de ser abordado é o da própria regulamentação da prática dos serviços de psicologia on-line. Os profissionais demonstraram estar de acordo com as diretrizes estabelecidas pela lei 012/2005 (CFP, 2005) que regulariza os atendimentos psicológicos mediados pelo computador. É importante considerar, entretanto, o viés de desejabilidade social implicada nas respostas dos profissionais, o que poderia tê-los levado a responder de acordo com o que seria esperado deles e não necessariamente a realidade da prática, que pode ser mais flexível e eventualmente não cumprir os padrões estabelecidos.

Sobre os pontos positivos, a possibilidade de anonimato foi um dos aspectos centrais relatado pelos participantes. Segundo Turkle (1997), esse é um dos mais relevantes aspectos das relações virtuais. $\mathrm{O}$ anonimato proporciona que os clientes se sintam mais à vontade, diminuindo a inibição, a autocensura, e aumentando a sinceridade, a espontaneidade e as probabilidades de mudança (Prado, 2000). Da mesma forma, Farah (2004) também enfatiza a questão do anonimato como um dos pontos principais pelos quais as pessoas buscam atendimento pela internet, evitando, assim, um estigma popular. Outros aspectos também foram citados na presente pesquisa e encontram fundamentação na literatura (Prado, 2000), tais como o fato de que o atendimento on-line propicia maior facilidade espacial e temporal, possibilidade de atender pessoas que estão viajando ou que não conhecem profissionais na sua região.

Em relação aos pontos negativos, alguns dos principais fatores relatados são o caráter impessoal/ superficial que algumas vezes o atendimento assume, a dificuldade de expressar emoções, a impossibilidade de avaliar o indivíduo de forma mais completa, por consequência da falta da interação corporal, por exemplo. Esse aspecto foi referido por Prado (2000), embora seu estudo também apresente alguns contra-argumentos em relação a isso, como a possibilidade de a emoção ser expressa por emoticons, imagens ou outros estímulos visuais, além de que os silêncios e respostas podem ser avaliados do ponto de vista comportamental. Corroborando esse argumento, outros autores também afirmam que, algumas vezes, é possível "sentir" o estado emocional da pessoa que escreveu através de sinais na escrita (Farah, 2004; Ohl, 2004), ou reforçando essa compreensão através da experiência sistemática com atendimentos e orientações on-line, gerando, assim, certo "aprendizado" para a leitura psicológica dos chats e trocas virtuais. A limitação para determinados tipos de caso, referida pelos profissionais que participaram deste estudo, também foi encontrada na 
literatura. Casos de pessoas com psicose ou em episódio maníaco, com necessidade de medicação ou monitoramento, com tendência à violência contra si ou contra outras pessoas e pessoas que abusam de substâncias são todas contraindicadas para atendimento on-line (Prado, 2000). A justificativa é de que nesses casos o profissional teria pouco controle e recursos para trabalhar com esses clientes no caso de uma eventual crise.

No que se refere aos questionamentos acerca do estabelecimento de vínculo, as respostas foram diversas. Alguns consideram o vínculo virtual bastante semelhante ao presencial, pois continua sendo uma relação na qual o cliente procura auxílio e o profissional busca ajudá-lo. Outros o consideram essencialmente diferente, uma vez que o tipo de contato é distinto e, portanto, a relação é outra. Entretanto, os entrevistados foram unânimes em afirmar que é possível estabelecer um vínculo adequado com o cliente, corroborando os resultados encontrados por Prado e Meyer (2006) em sua pesquisa sobre terapia virtual assíncrona, na qual concluíram que é possível estabelecer, via internet, um clima agradável e produtivo entre pacientes e profissionais. Spaccaquerche (2005) reforça essa hipótese na conclusão de seu estudo sobre orientação profissional. Os profissionais também concordaram em que é possível auxiliar o cliente na sua demanda, ainda que seja de uma forma limitada. É válido observar que a atividade dos profissionais é a orientação psicológica, não a terapia on-line, e esse pode ser o motivo da adjetivação do atendimento on-line como menos intenso e menos profundo, conforme trazida por eles.

Os fatores determinantes para o desenvolvimento do atendimento on-line apontados pelos entrevistados também possuem fundamentação teórica, especialmente as variáveis do terapeuta. Segundo autores que escreveram e estudaram a relação terapêutica (Beck et al., 1997; Freeman \& Dattilio, 1998; Meyer \& Vermes, 2001; Rogers, 1975, 1997; Washton \& Zweben, 2009), o terapeuta é responsável por estimular o vínculo saudável e produtivo com o cliente/paciente, e de suas características e capacidades de estabelecer essa relação depende, em grande parte, o sucesso ou fracasso do atendimento. Entretanto, como essa relação depende da aceitação e cooperação dos dois lados, o cliente também deve estar disposto a se vincular. Os profissionais entrevistados atribuem grande valor à disposição de o cliente fazer esse vínculo, ou seja, para os participantes, a motivação do indivíduo tem um papel determinante na relação positiva entre profissional e cliente. Esse fato levanta a discussão de que talvez no atendimento pela internet a motivação do paciente/cliente possa ser ainda mais importante, determinando diretamente o alcance dos objetivos.

Em contrapartida, uma pesquisa realizada com dois grupos (Cavalcante, 2005), um constituído por psicólogos que fazem atendimentos pela internet e outro composto por psicólogos que não o fazem, demonstrou que os psicólogos que não realizam atendimentos on-line são desfavoráveis a essa prática. O contato limitado e não presencial foi um dos motivos principais para essa posição contrária por parte dos entrevistados. Esses profissionais que não realizam atendimentos on-line afirmaram que seria inviável um atendimento adequado sem contato presencial, pois, assim, não se estabelece uma relação genuína, que é sua principal ferramenta. Entretanto, esses mesmos profissionais verbalizaram não ter conhecimento consistente a respeito dos serviços mediados pelo computador, tampouco da legislação pertinente. Desse modo, pode-se refletir a respeito desse tema: se, por um lado, existe oposição à realização de atendimentos via internet, por outro lado talvez seja necessário explorar melhor esse campo de atuação, para que se possam fazer críticas melhor fundamentadas.

Outros fatores também foram apontados pelos profissionais da presente pesquisa como entraves para o estabelecimento do vínculo em atendimentos on-line, os quais precisam ser discutidos. A desconfiança em relação a serviços oferecidos na internet, a familiaridade (ou falta dela) com a tecnologia e a preferência pelo atendimento tradicional que, como já vimos, é o mais presente na visão que as pessoas têm do psicólogo, são alguns desses fatores.

Diversos autores também salientam o papel da empatia na relação do terapeuta e seu paciente (Beck et al., 1997; Beck, 1997; Meyer \& Vermes, 2001; Rogers, 1975; Safran, 2002). 
Rogers (1975), por exemplo, definiu que a forma como o paciente percebe a consulta é um fator essencial para o atendimento. Contemplando essa discussão, na categoria Expressão de empatia, os participantes afirmaram considerar muito importante transmitir empatia aos clientes. Todavia, um aspecto diferencial do atendimento pela internet é a forma pela qual ele é feito. Os profissionais relataram que, por se tratar de um atendimento online, muitas vezes precisam lançar mão de outras técnicas para conseguir expressar essa empatia. Ou seja, a linguagem escrita precisa ser mais objetiva, clara, concreta, a fim de fazer com que o paciente entenda o que o profissional está querendo passar. Baldanza (2006), afirma que o corpo é essencial nas relações entre seres humanos e que ele não deixa de estar presente, mesmo no contexto virtual: a pontuação, letras maiúsculas, repetição de letras, todos esses recursos visam expressar algo que o corpo está vivenciando. Da mesma forma, às vezes também é difícil entender e sentir o que o cliente está falando ou sentindo, sendo necessário obter feedback mais frequentemente e fazer mais perguntas.

Farah (2004) comenta a questão, dizendo que somos treinados para a escuta na clínica tradicional. Entretanto, para os atendimentos pela internet faz-se necessário treinar uma nova forma de escuta. A maneira como o texto é escrito, as palavras e frases usadas, esse conjunto de elementos transmite alguma coisa. Ou seja, através de uma forma específica de análise é possível sentir as emoções e sentimentos presentes no texto. Contudo, ainda não possuímos, no âmbito dos serviços mediados por computador, parâmetros para discernir a subjetividade do profissional na interpretação das mensagens. Assim, os profissionais devem trabalhar com as suas próprias impressões, sem se deixar levar apenas por elas (Farah, 2004).

Não obstante, é interessante o relato dos participantes de que seus clientes verbalizam sentir o profissional ao seu lado, mesmo à distância, fenômeno também apontado por Prado (2000) em uma revisão de literatura. Para Turkle (1997), o anonimato propicia uma sensação de amparo. Dessa forma, o indivíduo que é atendido pela internet pode ter o sentimento de acolhimento potencializado por essa condição, na qual não há julgamentos sociais e preconceitos (Baldanza, 2006).

Na categoria Resposta a objetivos observa-se que os profissionais, a partir dos relatos dos seus clientes, avaliam que conseguem atingir os objetivos propostos nas orientações psicológicas on-line. Em alguns casos, porém, o objetivo é apenas avaliar e encaminhar a pessoa para o atendimento presencial, o que também pode ser considerado um fator de êxito, visto que constitui uma mudança importante na vida do sujeito e é apontado por Fortim e Cosentino (2007) como uma das funções e pontos positivos da orientação on-line.

\section{Considerações finais}

A orientação psicológica on-line constitui prática até recentemente inexplorada. O presente estudo objetivou compreender melhor essa prática a partir da percepção dos profissionais, especialmente no que diz respeito à relação dos profissionais com os clientes. Sendo assim, a presente pesquisa concluiu seus objetivos. Apesar disso, é necessário frisar que este é um estudo com limitações metodológicas. Outras pesquisas, de diferentes abordagens, qualitativas e quantitativas, deverão investigar aspectos específicos dos atendimentos on-line, como a relação terapêutica e efetividade dos tratamentos. Pesquisas posteriores também poderão incluir a percepção dos pacientes, o que não foi possível no presente estudo.

Com base no conteúdo das entrevistas pode-se pensar que talvez seja possível, nas consultas psicológicas pela internet, desenvolver uma relação positiva com os clientes, expressar empatia e alcançar objetivos. Contudo, para tanto, é necessário adaptar-se a esse novo campo, que possui linguagem e expressão próprias. Além disso, é importante considerar os aspectos positivos e negativos levantados pelos participantes, bem como as características específicas do fazer desses profissionais.

É incontestável a afirmação de que as novas tecnologias vieram para ficar. Entretanto, não se pode adotar, como refere Marcondes Filho (2001), nem uma posição de "aceitação entusiasta" de toda nova tecnologia, nem a de "refutação imediata" de toda e qualquer forma de inovação tecnológica. Para Lévy (2008), a 
única forma de desenvolver a utilização das novas tecnologias numa perspectiva humanista é nos mantermos receptivos em relação à novidade, tentando verdadeiramente compreendê-la, pois não se trata de ser contra ou a favor e sim de reconhecer as inevitáveis transformações culturais advindas da tecnologia. Da mesma forma, afirmam Almeida e Rodrigues (2003) que se pensarmos a clínica formalmente certamente concluiremos que um atendimento terapêutico à distância não é possível, pois não há a presença física do terapeuta, a relação face a face considerada fundamental. Todavia, a psicoterapia tradicional tem sido reinventada desde seus primórdios de acordo com mudanças no contexto psicossocial e pensar que está definido o modo correto de fazê-la pode representar um "fechamento" ao novo e às vantagens que as inovações têm a oferecer.

Ademais, é imprescindível que se fique atento, conforme refere Prado (2000), para a necessidade de que as pesquisas sejam desenvolvidas de forma adequada e para a contribuição que os atendimentos on-line poderiam dar para a Psicologia e para a compreensão do ser humano. A tecnologia está cada vez mais integrada ao nosso modo de vida e a Psicologia não pode se distanciar desse fenômeno. Além disso, deve-se, sobretudo, levar em conta aspectos éticos, entre eles, o preparo do profissional que decide atuar no campo virtual.

Portanto, cabe à Psicologia, como ciência, debruçar-se sobre esse campo da prática profissional e da dimensão humana. A interação entre Psicologia e informática, embora tenha poucos anos de existência, gradualmente deixa de parecer tão nova e se impõe, alheia às nossas discussões e vontades, trazendo ainda surpresas, desafios, dificuldades e potencialidades. Quanto mais pesquisas e esforços forem implementados para compreender e fundamentar a prática da Psicologia pela internet, maior poderá ser o preparo dos profissionais de Psicologia, seja para atendimentos clínicos presenciais, à distância, ou mesmo nas demais áreas da nossa profissão. É necessário que os psicólogos formados, e os atualmente em formação tenham uma visão mais ampla do sujeito contemporâneo. A internet já faz parte do nosso contexto social com tanta intensidade e força que sem o adequado entendimento de sua influência no psiquismo estar-se-á significativamente distante da própria realidade e das novas formas de subjetivação do mundo em que vivemos. 


\section{Gerson Siegmund}

Mestre em Psicologia pela Universidade Federal do Rio Grande do Sul, Porto Alegre - RS. Brasil. E-mail: peregrino.siegmund@gmail.com

\section{Carolina Lisboa}

Doutora em Psicologia pela Universidade Federal do Rio Grande do Sul, Porto Alegre - Brasil. Docente da Pontifícia Universidade Católica do Rio Grande do Sul, Porto Alegre - RS. Brasil.

E-mail: csmacedo@unisinos.br

Endereço para envio de correspondência:

Universidade do Vale do Rio dos Sinos. Av. Unisinos, 950. Bairro Cristo Rei. CEP 93022-000 São Leopoldo, RS. Brasil.

Recebido 11/09/2012, Aprovado 12/12/2013. 
Aboujaoude, E. (2011). Prefácio. In K. S. Young \& C. N. Abreu (Orgs.), Dependência de Internet: manual e guia de avaliação e tratamento (pp. 9-10). Porto Alegre: Artmed.

Almeida, L., \& Rodrigues, J. (2003). Narrativa e internet: possibilidade e limites do atendimento psicoterápico mediado pelo computador. Psicologia: Ciência e Profissão, 23(3), 10-17. Recuperado de http:// pepsic.bvsalud.org/scielo.php?pid=S1414$98932003000300003 \&$ script $=$ sci arttext\&tlng $=$ en

Baldanza, R. F. (2006). A comunicação no Ciberespaço: reflexões sobre a relação do corpo na interação e sociabilidade em espaço virtual. In VI Encontro dos Núcleos de Pesquisa da Intercom. Brasília: Núcleo de Pesquisa em Tecnologias da Informação e da Comunicação. Recuperado de http://galaxy.intercom.org. br:8180/dspace/bitstream/1904/20253/1/ Renata+Francisco+Baldanza.pdf

Bardin, L. (1995). Análise de conteúdo. Lisboa: Edições 70.

Bastos, A. V. B., \& Gomide, P. I. C. (1989). O psicólogo brasileiro: sua atuação e formação profissional. Psicologia: Ciência e Profissão, 9(1), 6-15. Recuperado de http://pepsic.bvsalud.org/

Bastos, A. V. B., \& Gondim, S. M. G. (2010). O trabalho do psicólogo no Brasil. Porto Alegre: Artmed.

Beck, A. T., Rush, A. J., Shaw, B. F., \& Emery, G. (1997). Terapia cognitiva da depressão. Porto Alegre: Artmed.

Beck, J. S. (1997). Terapia cognitiva: teoria e prática. Porto Alegre: Artmed.

Bouchard, S., Paquin, B., Payeur, R., Allard, M., Rivard, V., Fournier, T., et al (2004). Delivering cognitive-behavior therapy for panic disorder with agoraphobia in videoconference. Telemedicine Journal and e-Health, 10(1), 13-25. http:// dx.doi.org/10.1089/153056204773644535. PMid:15104911

Cavalcante, A. P. (2005). Terapia na rede: um estudo sobre a clínica mediada pelo computador na realidade brasileira. In: Conselho Regional de Psicologia de São Paulo, Psicologia e Informática: desenvolvimentos e progressos (pp. 157-174). São Paulo: Casa do Psicólogo.

Conselho Federal de Psicologia - CFP. (2005). Resolução CFP Nº 012/2005. Regulamenta o atendimento psicoterapêutico e outros serviços psicológicos mediados por computador e revoga a Resolução CFP № 003/2000. Recuperado de http://www.pol.org.br/pol/export/sites/ default/pol/legislacao/legislacaoDocumentos/ resolucao2005_12.pdf

Eizirik, C. L., Libermann, Z., \& Costa, F. (2008). A relação terapêutica: transferência, contratransferência, aliança terapêutica, e relação real. In: A. V. Cordioli (Org.), Psicoterapias: abordagens atuais (3. ed., pp. 67-75). Porto Alegre: Artmed.

Farah, R. M. (2004). Orientação psicológica via e-mail - serviço oferecido pela clínica psicológica da PUC-SP. In: R. M. Farah (Org.), Psicologia e Informática: o ser humano diante das novas tecnologias (p. 44-66). São Paulo: Oficina do Livro.

Fortim, I. F. (2004). Terapia on line e orientação psicológica: diferenças. In: R. M. Farah (Org.), Psicologia e Informática: o ser humano diante das novas tecnologias (pp. 43-47). São Paulo: Oficina do Livro.

Fortim, I. F., \& Cosentino, L. A. M. (2007). Serviço de orientação via e-mail: novas considerações. Psicologia: Ciência e Profissão, 27(1), 164-175. Recuperado de http://pepsic.bvsalud.org

Freeman, A., \& Dattilio, F. M. (1998). Compreendendo a terapia cognitiva. São Paulo: Psy.

Gondim, S. M. G., Bastos, A. V. B., \& Peixoto, L. S. A. (2010). Áreas de atuação, atividades e abordagens teóricas do psicólogo brasileiro. In A. V. B. Bastos \& S. M. G. Gondim (Orgs.), O trabalho do psicólogo no Brasil (pp. 174-199). Porto Alegre: Artmed.

Heloani, R., Macêdo, K. B., \& Cassiolato, R. (2010). O exercício da profissão: características gerais da inserção profissional do psicólogo. In A. V. B. Bastos \& S. M. G. Gondim (Orgs.), O trabalho do psicólogo no Brasil (pp. 107-130). Porto Alegre: Artmed.

Instituto Brasileiro de Opinião Pública e Estatística - IBOPE. (2011). Pesquisas. Recuperado de http://www.ibope.com.br/calandraWeb/servlet/ CalandraRedirect?temp $=6 \&$ proj $=$ PortalIBOP $\mathrm{E} \& \mathrm{pub}=\mathrm{T} \& \mathrm{db}=\mathrm{caldb} \& \mathrm{comp}=$ pesquisa_leitu ra\&nivel $=$ null \&docid $=$ C2A2CAE41B62E75E $83257907000 \mathrm{ECO4F}$

Lévy, P. (2008). Cibercultura (2. ed.). São Paulo: Editora 34. (Trabalho original publicado em 1997). 
Magalhães, M., Straliotto, M., Keller, M., \& Gomes, W. B. (2001). Eu quero ajudar as pessoas: a escolha vocacional da psicologia. Psicologia: Ciência e Profissão, 21(2), 10-27. Retrieved from http://pepsic.bvsalud.org/

Malvezzi, S. (2010). A profissionalização dos psicólogos: uma história de promoção humana. In A. V. B. Bastos \& S. M. G. Gondim (Orgs.), O trabalho do psicólogo no Brasil (pp. 17-31). Porto Alegre: Artmed.

Marcondes Filho, C. (2001). Haverá vida após a internet? Famecos, 16(1), 35-45. Recuperado de http://revistaseletronicas.pucrs.br

Meyer, S., \& Vermes, J. (2001). Relação terapêutica. In B. Rangé (Org.), Psicoterapias cognitivocomportamentais: um diálogo com a psiquiatria (pp. 101-110). Porto Alegre: Artmed.

Nicolaci-da-Costa, A. M. (2005). Internet e subjetividade: a emergência de uma nova "configuração psíquica". In Conselho Regional de Psicologia de São Paulo, Psicologia e Informática: Desenvolvimentos e progressos (pp. 11-29). São Paulo: Casa do Psicólogo.

Ohl, G. T. (2004). Algumas diferenciações entre orientação psicológica mediada por computadores e a "psicologia de revista". In R. M. Farah (Org.), Psicologia e Informática: o ser humano diante das novas tecnologias (p. 213-214). São Paulo: Oficina do Livro.

Organização das Nações Unidas - ONU. (2009). Manual for measuring ICT access and use by households and individuals. Recuperado de http://www.uneca.org/aisi/scanict/training/ ITU_Manual.pdf

Organização das Nações Unidas - ONU. (2011). Internet users \%. Recuperado de http://data. un.org/

Prado, O. Z. (2000). Terapia on-line: aspectos da ética, sua metodologia e as potencialidades e restrições. In E. Sayeg (Org.), Psicologia e informática: interfaces e desafios (pp. 75-103). São Paulo: Casa do Psicólogo.
Prado, O. Z., \& Meyer, S. B. (2006). Avaliação da relação terapêutica na terapia assíncrona via internet. Psicologia em Estudo, 11(2), 247-257. http://dx.doi.org/10.1590/S141373722006000200003.

Rogers, C. R. (1975). A terapia centrada no paciente. São Paulo: Martins Fontes. (Trabalho original publicado em 1958).

Rogers, C. R. (1997). Psicoterapia e consulta psicológica (2. ed.). São Paulo: Martins Fontes. (Trabalho original publicado em 1969).

Safran, J. D. (2002). Ampliando os limites da terapia cognitiva: o relacionamento terapêutico, a emoção e o processo de mudança. Porto Alegre: Artmed.

Spaccaquerche, M. E. (2005). Orientação profissional online: uma experiência em processo. Revista Brasileira de Orientação Profissional, 6 (1), 6374. Recuperado de http://pepsic.bvsalud.org

Spek, V., Cuijpers, P., Nyklícek, I., Riper, H., Keyzer, J., \& Pop, V. (2007). Internet-based cognitive behaviour therapy for symptoms of depression and anxiety: a meta-analysis. Psychological Medicine, 37(3), 319-328. http:// dx.doi.org/10.1017/S0033291706008944. PMid:17112400

Turkle, S. (1997). Life on the screen: identity in the age of the internet. New York: Touchstone.

van Bastelaar, K., Cuijpers, P., Pouwer, F., Riper, H., \& Snoek, F. J. (2011). Development and reach of a web-based cognitive behavioural therapy programme to reduce symptoms of depression and diabetes-specific distress. Patient Education and Counseling, 84(1), 49-55. http://dx.doi.org/10.1016/j.pec.2010.06.013. PMid:20619577

Washton, A. M., \& Zweben, J. E. (2009). Prática psicoterápica eficaz dos problemas com álcool e drogas. Porto Alegre: Artmed.

Young, K. S., \& Abreu, C. N. (Orgs.). (2011). Dependência de Internet: manual e guia de avaliação e tratamento. Porto Alegre: Artmed. 